\title{
Assessment of Genetic Diversity and Fingerprinting of Rice Cultivars of Andhra Pradesh and Telangana with SSR Markers
}

\author{
Balram Marathi* and Maliha Allugundu \\ Institute of Biotechnology, Professor Jayashankar Telangana State Agricultural University, \\ Rajendranagar, Hyderabad - 500030, India \\ *Corresponding author
}

\begin{abstract}
Keywords
Rice, SSR markers, Genetic diversity, DNA

Fingerprinting, Andhra Pradesh, Telangana

Article Info

Accepted:

17 November 2018 Available Online:

10 December 2018 markers were polymorphic among 53 varieties. A total of 55 alleles were produced for 23 markers with fragment size ranging from 60bp (RM204) to 470bp (RGNMS1289). The number of alleles per marker were ranged from two (RM262) to four (RGNMS1776) with an average of 2.39 alleles/loci. The PIC value for this set of markers varied from 0.213 (RM5474) to 0.670 (RM152), while the gene diversity ranged from 0.228 (RM5474) to 0.720 (RM152). Cluster analysis revealed a total of four distinct clusters resulted out of analysis of pooled SSR marker data. First cluster contains eleven varieties, most of them released from ARI, Rajendranagar, Hyderabad. Second cluster was further divided into two sub-clusters, the varieties grouped into cluster IIa commonly had Mahsuri as one of the parents and cluster IIb had mostly varieties released from Maruteru and Warangal. The cluster III contained seven varieties, most of them developed from Maruteru and cluster IV had only two varieties i.e., Sumati and Nellore Mahsuri having a common character of very long slender grain type. Four SSR loci produced variety specific unique alleles for five varieties, which will be useful in in identification of these varieties. The results in the present study will be useful in understanding molecular variability of the varieties developed and in diversifying the genetic base of rice breeding program, identification of rice varieties in protection of IPR.
\end{abstract}

\section{A B S T R A C T}

Twenty three simple sequence repeat markers were used to study genetic relationship among fifty three rice varieties developed from Andhra Pradesh and Telangana states. All

\section{Introduction}

Green revolution, mainly centered on high yielding varieties, has revolutionized rice production since the late 1960's. These varieties are characterized by higher yield potential, better grain quality, shorter growth duration, multiple resistance to diseases and insects and tolerance to problem soils. High yielding varieties are now planted in $70 \%$ of the world's rice lands (Khush, 1995). In India, to date 1,175 high yielding varieties have been released suitable for different ecologies (Shobha Rani et al., 2014; DARE Annual Report, 2017-18). Rice was cultivated in an area of 21.6 lakh hectares and 10.46 lakh hectares in Andhra Pradesh and Telangana respectively during 2015-16 (DES, AP, 2017; DES, TS, 2017). Until the year 2014, approximately 55 and 34 high yielding rice 
varieties were released from Andhra Pradesh and Telangana respectively (Shobha Rani et al., 2014). A detailed understanding of the extent and structure of genetic diversity is necessary for effective management and use of crop germplasm resources. DNA-based markers such as SSR markers (or microsatellites), which are codominant and highly polymorphic, offer an easy, accurate, and quantifiable measure of the genetic variation within crop species (Litt and Luty, 1989; Tautz, 1989). For rice, nearly 52, 485 SSRs were developed by Zhang et al., 2007 and these are being used to develop highdensity genetic maps, genotype rice accessions, determine the genetic structure and diversity patterns, optimize the assembly of core collections, and for marker-assisted breeding (McCouch et al., 2002; Yu et al., 2003; Garris et al., 2005). Apart from SSRs, around 19,555 rice Genic non-coding microsatellite (RGNMS) were developed and reported by Parida et al., (2009). Even though studies to assess the genetic diversity within $O$. sativa collections utilizing isozyme, restriction fragment length polymorphism (RFLP) and SSR markers have been conducted (Glaszmann, 1987; Yang et al., 1994; Xu et al., 2004; Lu et al., 2005; Garris et al., 2005, Chakravarthi et al., 2006) there has been no comprehensive report on genetic diversity among varieties of Andhra Pradesh and Telangana at the DNA level. The objectives of this study were to use SSR markers to (i) quantify the allelic diversity, (ii) estimate the genetic diversity, and (iii) DNA fingerprinting of 53 rice cultivars including released and advance breeding material from different research institutes of Andhra Pradesh and Telangana.

\section{Materials and Methods}

\section{Plant material}

Fifty three rice cultivars (22 from Andhra Pradesh and 31 from Telangana) that include released varieties and advanced breeding material were selected for SSR screening (Table 1). This material includes rice varieties released and under minikit trials from different research stations of Andhra Pradesh and Telangana which are having special attributes like photoinsensitivity, quality, resistance to different pests and diseases. Seeds were kindly provided by seven main rice breeding stations of Andhra Pradesh and Telangana, which include Andhra Pradesh Rice Research Institute, Maruteru, Agricultural Reserch Institute, Hyderabad, Regional Agricultural Research Station, Warangal, Regional Agricultural Research Station, Jagtial, Regional Sugarcane and Rice Research Station, Rudrur, Agricultural Research Station, Bapatla and Agricultural Research Station, Nellore.

\section{DNA extraction and SSR analysis}

Plants were grown in a growth chamber under controlled conditions of light and temperature. Leaves of 15 days old seedlings were collected, frozen in liquid $\mathrm{N}_{2}$, and stored at $-80^{\circ} \mathrm{C}$ until used. DNA was isolated by CTAB (Cetyl- Tetra Methyl Ammonium Bromide) method (Murray and Thompson, 1980). Twenty-three SSR markers includng Rice Microsatellite (RM) markers and rice Genic noncoding microsatellite (RGNMS) markers linked to nine yield attributing traits representing the six of the twelve rice chromosomes, were used for genotyping (Table 2). PCR products were separated on a $3.5 \%$ Metapphor gels and DNA fragments were visualized using Gel star stain (BMA, USA). The size of the amplified fragments was determined by measuring migration distances of SSR alleles on gel photographs in relation to known fragment-length standard 50bp ladder (MBI Fermentas, Lithuania) which was run alongside the samples. When a null allele was detected, the result was confirmed by two independent PCR amplification reactions. Unique alleles were 
defined as those detected in only one accession.

\section{Data analysis}

All the genotypes were scored for the presence and absence of the SSR bands. The data was entered into a binary matrix as discrete variables, 1 for presence and 0 for absence of the character and this data matrix was subjected to further analysis. The Excel file containing the binary data was imported into NT Edit of NTSYS v. 2.02 (Rohlf, 1998). The 0/1 matrix was used to calculate Similarity as DICE coefficient using SIMQUAL subroutine in SIMILARITY routine. The resultant similarity matrix was employed to construct dendrograms using Sequential Agglomerative Hierarchical Nesting (SAHN) based Unweighted Pair Group Method with Arithmetic Means (UPGMA) to infer genetic relationships and phylogeny. In addition, GGT 2.0 (Berloo, 2008) was used to visualize chromosomal maps of 53 varieties with genotypic information of 23 SSR markers.

\section{Results and Discussion}

Assessment of genetic diversity is important for better utlization of breeding material. Results obtained in genetic diversity studies of $O$. sativa cultivars with RFLP and RAPD markers indicate that more genetic diversity exists in indica and japonica gene pools (Fuentes et al., 1999; Qian et al., 1995). Classical breeding affects genetic diversity within breeding programs. Selection increases the frequency of alleles or allelic combinations with favorable effects at the expense of others, eventually eliminating many of them (Cao et al., 1998). In the present investigation, microsatellites (Rice microsatellites) or SSR markers (Simple Sequence Repeats) from chromosome numbers $2,3,4,5,6$ and 8 were used to characterize and to assess genetic diversity among 53 rice cultures. A total of 23 microsatellite primers including 16 RM series primers and 7 RGNMS primers were utilized. All 23 primers showed polymorphism between 53 rice cultivars. A total of 55 alleles were scored with 23 markers for a set of 53 varieties where the allele size ranged from 60bp (RM204) to 470bp (RGNMS1289). The study revealed that the primer RGNMS1776 had maximum number (four) of alleles followed by three alleles each for RM3698, RM16，RM251，RM5709，RM431，RM190 and RM152, while rest of the fifteen markers had two alleles each, with the average being 2.39 alleles and the allele frequency ranged from 0.01 to 0.85 . The PIC value for this set of markers varied from 0.213 (RM5474) to 0.670 (RM152), while the gene diversity ranged from 0.228 (RM5474) to 0.720 (RM152) (Table 2). A representative gel picture showing the banding pattern of these varieties with the markers has been depicted in Figure 1. Similar observations were made by Akagi et al., (1997) that RM1 and RM3 and four other RM markers were less polymorphic. Many studies have also reported significantly greater allelic diversity of microsatellite markers than other molecular markers (McCouch et al., 2001).

Cluster analysis was used to group the varieties and to construct a dendrogram. The similarity matrix representing the DICE Coefficient was used to cluster the data using the UPGMA algorithm. The UPGMA based dendrogram obtained from the binary data deduced from the DNA profiles of the samples analyzed adds a new dimension to the genetic similarity perspectives generated. A total of four distinct clusters resulted out of analysis of pooled SSR marker data (Fig. 2). This dendrogram revealed that the genotypes that are derivatives of genetically similar type clustered more together, i.e., the varieties derived from crosses having common parents were grouped together in a cluster thus reducing their diversity (Deepti et al., 2011; Gangaprasad et al., 2013). 
Table.1 Information on pedigree, source station and status of genetic material

\begin{tabular}{|c|c|c|c|c|}
\hline S.No & Name & Pedigree & Source Research Station & Year \\
\hline 1 & Swarna & Vasistha/Mahsuri & A.P.R.R.I, Maruteru, Andhra Pradesh & 1982 \\
\hline 2 & Prabhat & IR 8/MTU 3 & A.P.R.R.I, Maruteru, Andhra Pradesh & 1976 \\
\hline 3 & Vajram & MTU 4569/ARC 6650 & A.P.R.R.I, Maruteru, Andhra Pradesh & 1986 \\
\hline 4 & Chaitanya & Sowbhagya/ARC 5984 & A.P.R.R.I, Maruteru, Andhra Pradesh & 1988 \\
\hline 5 & Krishnaveni & Sowbhagya/ARC 5984 & A.P.R.R.I, Maruteru, Andhra Pradesh & 1989 \\
\hline 6 & Nandi & Sowbhagya/ARC 6650 & A.P.R.R.I, Maruteru, Andhra Pradesh & 1991 \\
\hline 7 & Vijetha & MTU 5249/MTU 7014 & A.P.R.R.I, Maruteru, Andhra Pradesh & 1995 \\
\hline 8 & Cottondora Sannalu & Krishnaveni/IR 64 & A.P.R.R.I, Maruteru, Andhra Pradesh & 2000 \\
\hline 9 & Indra (MTU1061) & PLA1100/MTU1010 & A.P.R.R.I, Maruteru, Andhra Pradesh & 2006 \\
\hline 11 & Amara (MTU1064) & PLA1100/MTU1010 & A.P.R.R.I, Maruteru, Andhra Pradesh & 2009 \\
\hline 10 & Pushyami (MTU1075) & MTU2716/MTU1010 & A.P.R.R.I, Maruteru, Andhra Pradesh & 2008 \\
\hline 12 & MTU1081 & BPT5204/Ajaya & A.P.R.R.I, Maruteru, Andhra Pradesh & Adv material \\
\hline 13 & Swarnamukhi & Cica 4 / IR 625-23-3-1//Tetep & A.R.S, Nellore, Andhra Pradesh & 1991 \\
\hline 14 & Sriranga & RP 5-32/Mahsuri & A.R.S, Nellore, Andhra Pradesh & 1991 \\
\hline 15 & Parthiva (NLR33892) & NLR27999/MTU4870 & A.R.S, Nellore, Andhra Pradesh & 2006 \\
\hline 16 & Bharani (NLR30491) & IR36/IET2508 & A.R.S, Nellore, Andhra Pradesh & 1997 \\
\hline 17 & Nellore Mahsuri (NLR34449) & IR72/BPT5204 & A.R.S, Nellore, Andhra Pradesh & 2009 \\
\hline 18 & Sona Mahsuri & Sona/Mahsuri & A.R.S. Bapatla, Andhra Pradesh & 1982 \\
\hline 19 & Samba Mahsuri & GEB 24/TN1//Mahsuri & A.R.S. Bapatla, Andhra Pradesh & 1986 \\
\hline 20 & Bapatla sannalu (BPT1768) & BPT3301/Mahsuri & A.R.S. Bapatla, Andhra Pradesh & 2001 \\
\hline 21 & Akshaya (BPT2231) & Surya/BPT6844 & A.R.S. Bapatla, Andhra Pradesh & 2010 \\
\hline 22 & Bhavapuri sannalu(BPT2270 & BPT5204/CR 15 MR 1523 & A.R.S. Bapatla, Andhra Pradesh & 2010 \\
\hline 23 & Tella Hamsa & HR 12/TN1 & ARI, R. Nagar, Hyderabad, Telangana & 1971 \\
\hline 24 & Rajendra & IJ 52/TN 1 & ARI, R. Nagar, Hyderabad, Telangana & 1976 \\
\hline 25 & Satya & Tella Hamsa/Rasi & ARI, R. Nagar, Hyderabad, Telangana & 1987 \\
\hline 26 & Saleem & GEB24/Sgadis//IR8/RNR8102 & ARI, R. Nagar, Hyderabad, Telangana & 1987 \\
\hline
\end{tabular}




\begin{tabular}{|c|c|c|c|c|}
\hline 27 & Chandana & Sona/Manoharsali & ARI, R. Nagar, Hyderabad, Telangana & 1989 \\
\hline 28 & Rajavadlu & Rajendra/IR30 & ARI, R. Nagar, Hyderabad, Telangana & 1993 \\
\hline 29 & Sagarsamba & IR8/Siam29//IR8/PTB21 & ARI, R. Nagar, Hyderabad, Telangana & 1993 \\
\hline 30 & Early samba (RNRM7) & Mutant of BPT5204 & ARI, R. Nagar, Hyderabad, Telangana & 2000 \\
\hline 31 & Sumati & Chandan / Pak. Basmati & ARI, R. Nagar, Hyderabad, Telangana & 2001 \\
\hline 32 & Pothana & IR 579/WGL 12708 & R.A.R.S, Warangal, Telangana & 1988 \\
\hline 33 & Surekha & IR 8/Siam 29 & R.A.R.S, Warangal, Telangana & 1976 \\
\hline 34 & Divya & WGL 23022/Surekha & R.A.R.S, Warangal, Telangana & 1989 \\
\hline 35 & Kavya & $\begin{array}{l}\text { WGL } 27120 \text { // Mahsuri // WGL } 17672 \text { // } \\
\text { Surekha }\end{array}$ & R.A.R.S, Warangal, Telangana & 1991 \\
\hline 36 & Erramallelu & BC 5-55/W 12708 & R.A.R.S, Warangal, Telangana & 1991 \\
\hline 37 & Orugallu & OBS 677/IR 2070-423-2-5 & R.A.R.S, Warangal, Telangana & 1993 \\
\hline 38 & Bhadrakali & Phalguna/IR 36 & R.A.R.S, Warangal, Telangana & 1994 \\
\hline 39 & Kesava & WGL 28712/IR 36-1996 & R.A.R.S, Warangal, Telangana & 1997 \\
\hline 40 & Siva & Phalguna/IR 50 & R.A.R.S, Warangal, Telangana & 1997 \\
\hline 41 & Varalu & WGL 20471 / CR 544-1-2 & R.A.R.S, Warangal, Telangana & 2001 \\
\hline 42 & Warangal Sannalu & Divya/BPT5204 & R.A.R.S, Warangal, Telangana & 2006 \\
\hline 43 & Warangal Samba & BPT5204/ARC5984//Sonamahsuri & R.A.R.S, Warangal, Telangana & 2000 \\
\hline 44 & Jagtial Samba (JGL3844) & BPT5204/ARC5984//kavya & RARS, Jagtial, Telangana & 2009 \\
\hline 45 & JGL13595 & MTU4870/JGL418 & RARS, Jagtial, Telangana & Adv material \\
\hline 46 & Pranahita (JGL11727) & JGL420/Vijetha & RARS, Jagtial, Telangana & 2012 \\
\hline 47 & Manair Sona (JGL3828) & BPT5204/Aganni & RARS, Jagtial, Telangana & 2009 \\
\hline 48 & Karimnagar Samba (JGL3855) & BPT5204/ARC5984//Kavya & RARS, Jagtial, Telangana & 2010 \\
\hline 49 & Jagtial Mahsuri (JGL11470) & JGL418/G.Beton & RARS, Jagtial, Telangana & 2010 \\
\hline 50 & Rudrama & HR19/TN1 & R.S and R.R.S, Rudrur, Telangana & 1991 \\
\hline 51 & Varsha & IR 50/Mahsuri & R.S and R.R.S, Rudrur, Telangana & 1993 \\
\hline 52 & Indursamba & BPT 5204/Surekha & R.S and R.R.S, Rudrur, Telangana & 1997 \\
\hline 53 & Pelalavadlu & OBS677/IR2070-423-2-5 & R.S and R.R.S, Rudrur, Telangana & 1998 \\
\hline
\end{tabular}


Table.2 Genetic variability parameters of 23 SSR markers

\begin{tabular}{|c|c|c|c|c|c|c|c|c|c|c|}
\hline \multirow[t]{2}{*}{ Sl. No } & \multirow[t]{2}{*}{ Chr. } & \multirow[t]{2}{*}{ Marker } & \multirow{2}{*}{$\begin{array}{l}\text { No. of } \\
\text { Alleles }\end{array}$} & \multicolumn{4}{|c|}{ Allele frequency } & \multirow[t]{2}{*}{ PIC } & \multirow{2}{*}{$\begin{array}{c}\text { Gene } \\
\text { Diversity }\end{array}$} & \multirow[t]{2}{*}{ Unique Alleles } \\
\hline & & & & 1 & 2 & 3 & 4 & & & \\
\hline 1 & 2 & RM262 & 2.0 & 0.406 & 0.594 & - & - & 0.366 & 0.482 & \\
\hline 2 & 2 & RGNMS3876 & 3.0 & 0.070 & 0.380 & 0.549 & - & 0.457 & 0.549 & Chandana $_{(330 \mathrm{bp})}$ \\
\hline 3 & 3 & RM7 & 2.0 & 0.571 & 0.429 & - & - & 0.370 & 0.490 & \\
\hline 4 & 3 & RGNMS 1140 & 2.0 & 0.341 & 0.659 & - & - & 0.348 & 0.449 & \\
\hline 5 & 3 & RM3698 & 3.0 & 0.230 & 0.690 & 0.080 & - & 0.407 & 0.464 & Indursamba $_{(200 \mathrm{bp})}$ \\
\hline 6 & 3 & RM16 & 3.0 & 0.121 & 0.712 & 0.167 & - & 0.407 & 0.450 & \\
\hline 7 & 3 & RM251 & 3.0 & 0.223 & 0.277 & 0.500 & - & 0.553 & 0.624 & \\
\hline 8 & 3 & RGNMS1289 & 2.0 & 0.526 & 0.474 & - & - & 0.374 & 0.499 & \\
\hline 9 & 3 & RM5474 & 3.0 & 0.873 & 0.093 & 0.034 & - & 0.213 & 0.228 & \\
\hline 10 & 4 & RM273 & 2.0 & 0.411 & 0.589 & - & - & 0.367 & 0.484 & \\
\hline 11 & 4 & RM241 & 3.0 & 0.128 & 0.855 & 0.017 & - & 0.228 & 0.253 & \\
\hline 12 & 4 & RGNMS1539 & 4.0 & 0.494 & 0.458 & 0.036 & 0.012 & 0.441 & 0.545 & \\
\hline 13 & 4 & RGNMS3276 & 2.0 & 0.626 & 0.374 & - & - & 0.359 & 0.468 & \\
\hline 14 & 4 & RM5709 & 3.0 & 0.275 & 0.681 & 0.044 & - & 0.386 & 0.458 & \\
\hline 15 & 4 & RM1112 & 2.0 & 0.419 & 0.581 & - & - & 0.368 & 0.487 & \\
\hline 16 & 5 & RGNMS1776 & 3.0 & 0.176 & 0.365 & 0.460 & - & 0.547 & 0.625 & \\
\hline 17 & 5 & RM413 & 4.0 & 0.270 & 0.617 & 0.061 & 0.052 & 0.478 & 0.540 & $\begin{array}{l}\text { Krishnaveni }_{(130 \mathrm{bp})} \text {, } \\
\text { Vajram }_{(120 \mathrm{bp})}\end{array}$ \\
\hline 18 & 6 & RM6273 & 2.0 & 0.675 & 0.325 & - & - & 0.343 & 0.439 & \\
\hline 19 & 6 & RM190 & 3.0 & 0.067 & 0.723 & 0.210 & - & 0.378 & 0.429 & \\
\hline 20 & 6 & RM204 & 4.0 & 0.337 & 0.495 & 0.059 & 0.109 & 0.560 & 0.626 & \\
\hline 21 & 6 & RGNMS3878 & 2.0 & 0.839 & 0.161 & - & - & 0.233 & 0.270 & Sona Mahsuri $_{(150 \mathrm{bp})}$ \\
\hline 22 & 8 & RM152 & 4.0 & 0.162 & 0.180 & 0.378 & 0.279 & 0.670 & 0.720 & \\
\hline 23 & 8 & RM25 & 3.0 & 0.046 & 0.282 & 0.673 & - & 0.392 & 0.466 & \\
\hline
\end{tabular}


Fig.1 Gel picture showing amplification pattern of RM413 in varieties

M 1234456678 9 101112131415161718192021222324252627282930313233343536373839404142434445464748

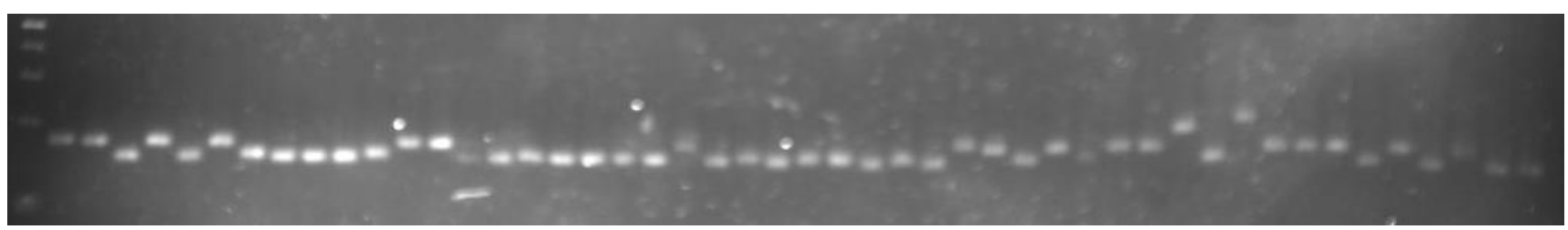

M- 100bp ladder; 1-48 - Variety numbers (Refer Table-1 for details)

Fig.2 Clustering of 53 Varieties of Rice based on 23 SSR markers

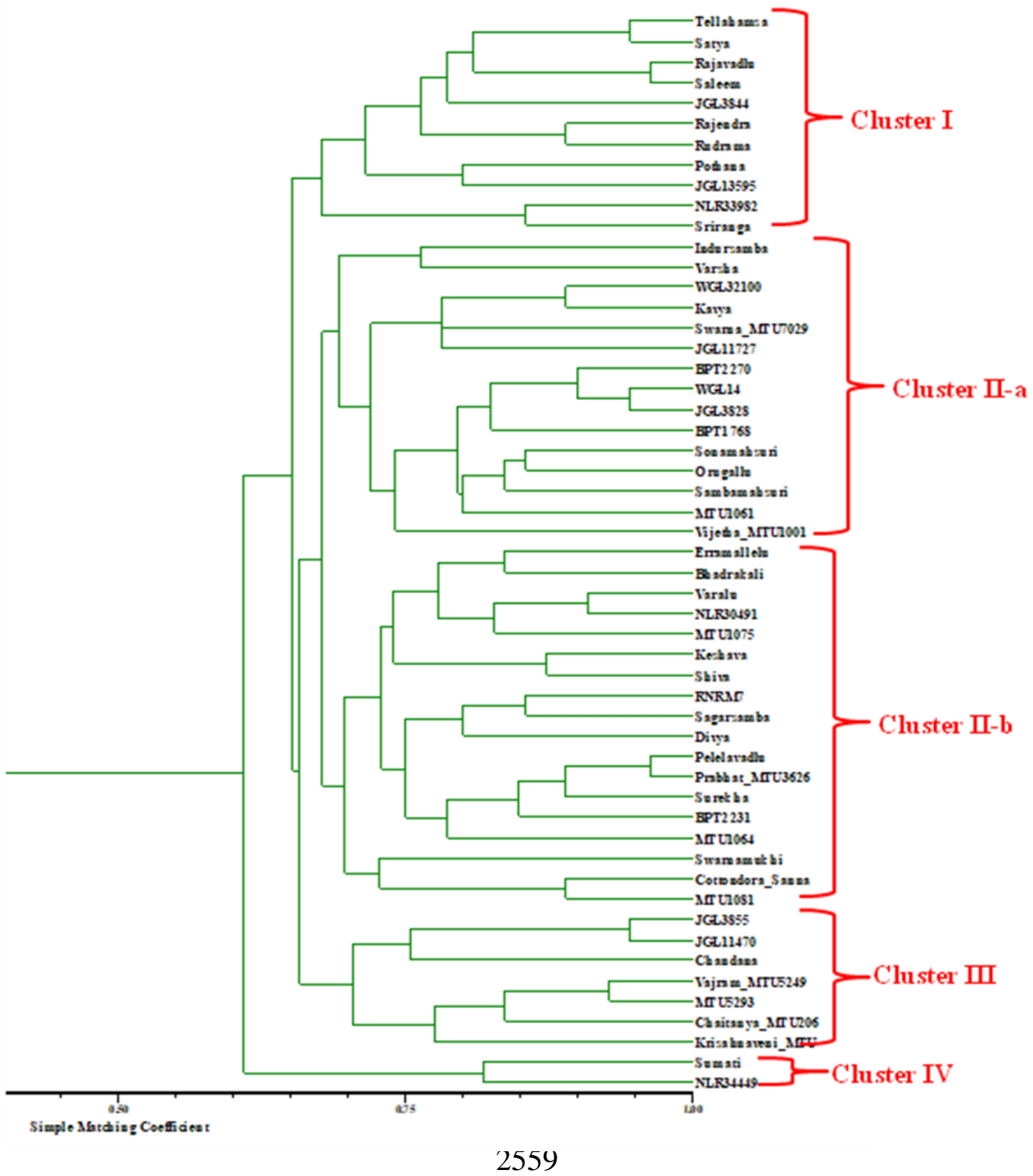


Fig.3 Graphical genotyping of 53 rice cultivars from Andhra Pradesh and Telangana based on 23 SSR markers

\begin{tabular}{l} 
Tellahamsa \\
Satya \\
Rajendra \\
Rudrama \\
Indursamba \\
Varsha \\
Erramallelu \\
Varalu \\
WGL32100 \\
Pothana \\
JGL3844 \\
JGL13595 \\
RNRM7 \\
Rajavadlu \\
Sumati \\
Saleem \\
NLR34449 \\
NLR33982 \\
NLR30491 \\
Swarnamukhi \\
Pelelavadlu \\
BPT2270 \\
BPT2231 \\
BPT1768 \\
Divya \\
Surekha \\
Bhadrakali \\
Kavya \\
WGL14 \\
JGL3828 \\
JGL3855 \\
JGL11470 \\
JGL11727 \\
Sagarsamba \\
Chandana \\
Sriranga \\
Sonamahsuri \\
Sambamahsuri \\
Keshava \\
Orugallu \\
Shiva \\
Swarna \\
Vajram \\
MTU5293 \\
Krisahnaveni \\
Chaitanya \\
MTU1075 \\
Prabhat \\
Vijetha \\
Cottondora Sannalu \\
MTU1061 \\
MTU1081 \\
MTU1064 \\
Consensus \\
\\
\hline Tha
\end{tabular}

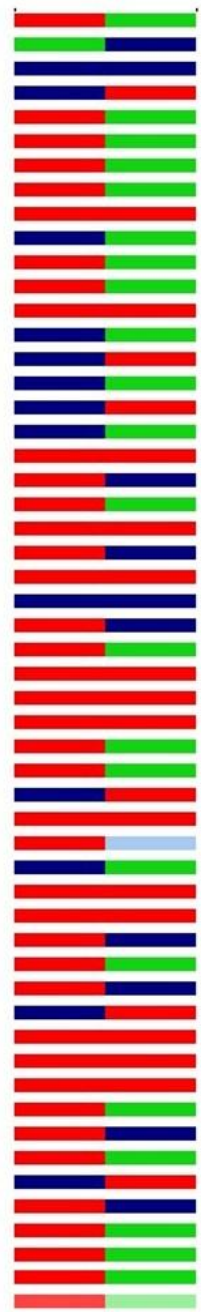

. . . . . .

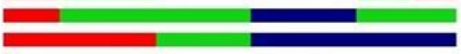

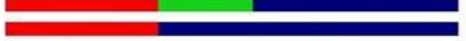

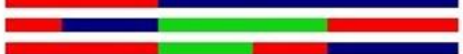

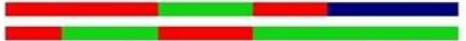

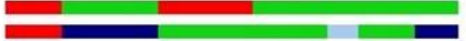
and a

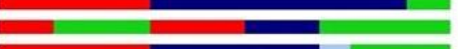
aㅏ

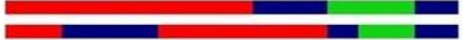

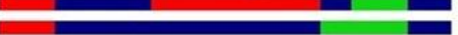

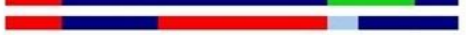
bar a

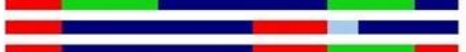

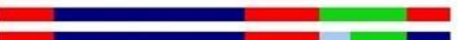
raras a

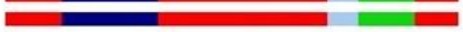
-

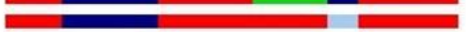
-

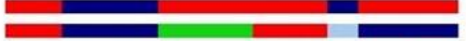

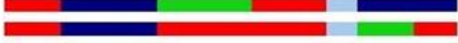

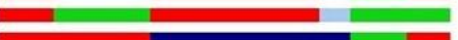

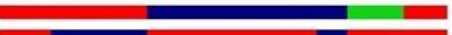

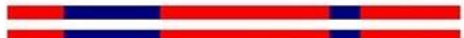

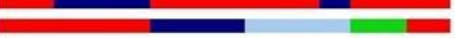
-

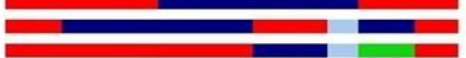

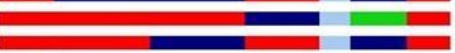

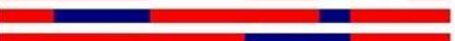

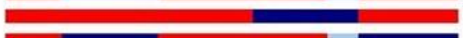

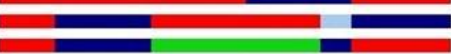

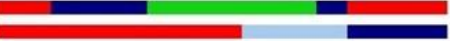

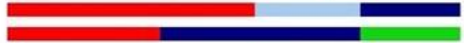

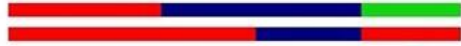

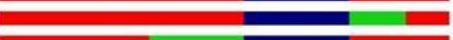

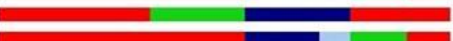

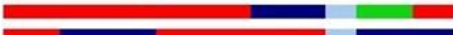
aras (a)

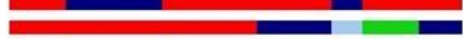

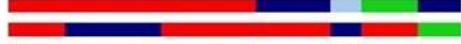

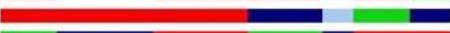

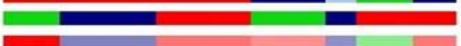

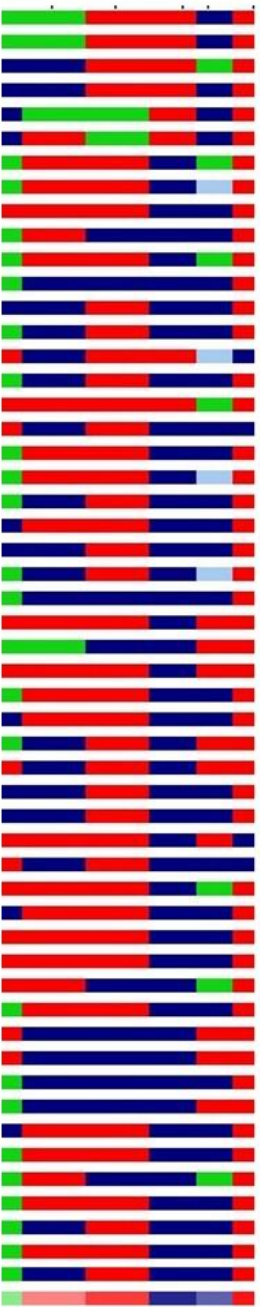

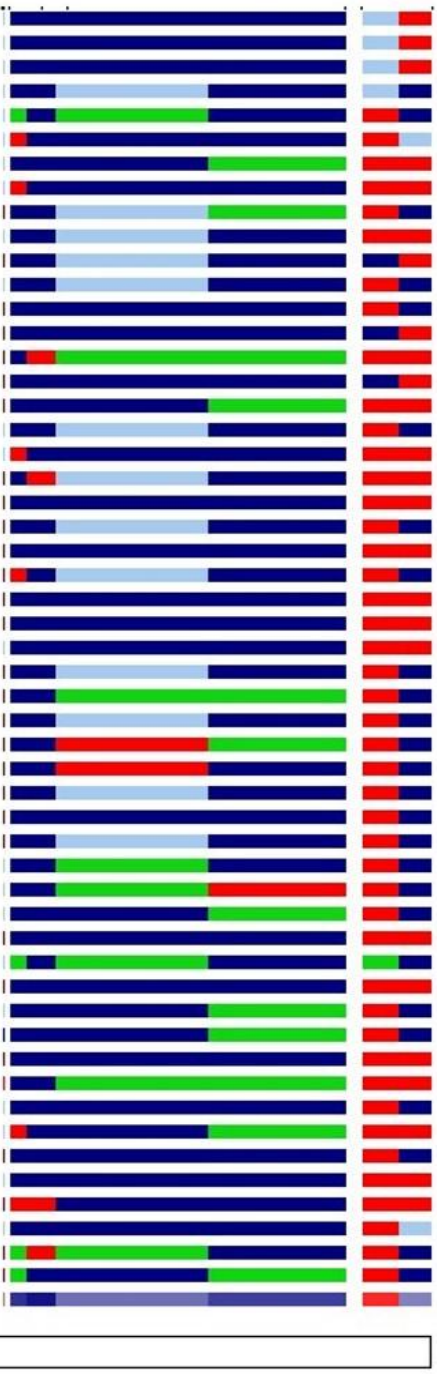

\section{Legend} B D

First cluster contains eleven varieties, most of them released from ARI, Rajendranagar, Hyderabad and others from Warangal, Jagtial, Rudrur and Nellore. The reason for these varieties clustering together might be their parentage. Second cluster was further seen to bifurcate into two sub-clusters containing fifteen and eighteen varieties respectively from all the research stations without a bias. It was observed that, the varieties grouped into cluster IIa commonly had Mahsuri as one of the parents, which might be the reason for these varieties to group together. On the other hand, cluster IIb had mostly varieties released from Maruteru and Warangal implying the repeated usage of same set of lines for crossing and release of new varieties. The cluster III contained seven varieties, most of them from Maruteru. Even in this cluster the parents used in crossing were the reason for grouping of varieties together. Finally, cluster IV had only two varieties (Sumati and Nellore Mahsuri) having a common character of very long slender grain type, which is a characteristic feature of Basmati type varieties. All the varieties are at least $65 \%$ 
similar to other meaning they are diverse for remaining $35 \%$ of the regions represented by the marker set used in the study.

Yu et al., 2003 carried out a similar study using 193 parental lines obtained from 26 countries and testing them with 110 well distributed SSR markers. An overall genetic diversity of 0.68 and an average of 6.3 alleles per locus were revealed, indicating a high level of genetic variation in these lines. Cluster analysis of the 193 accessions showed three major groups and nine subgroups. Group I corresponded to the classical indica subspecies, whereas groups II and III belong to the japonica subspecies.

Similar work was done on Indian scented and quality germplasm was done using fluorescently labeled SSR markers. A set of 69 rice varieties including 52 basmati and other quality varieties from different parts of India and 17 indica and japonica varieties that served as controls were used. These varieties were tested using a set of 30 SSRs as a result of which a total of 235 alleles were detected, of which $62(26.4 \%)$ were present only in Basmati and other scented rice germplasm accessions. The number of alleles per locus ranged from 3 to 22, with an average of 7.8 and polymorphism information content (PIC) values ranged from 0.2 to 0.9 , with an average of 0.6. Of the 30 SSR markers, 20 could distinguish traditional Basmati rice varieties, and a single panel of eight markers could be used to differentiate the premium traditional Basmati, cross-bred Basmati, and nonBasmati rice varieties having different commercial value in the marketplace. The results of this study indicated that Indian aromatic and quality germplasm is genetically distinct from other groups within $O$. sativa and is the product of a long, independent pattern of evolution (Jain et al., 2004).

In another study carried out by Spada et al., 2004, a set of 96 rice cultivars was tested using AFLP and SSR markers. AFLP produced 461 fragments, 248 (53\%) of which were polymorphic, SSR produced four to 11 alleles in the 12 genomic loci investigated. Both AFLP and SSR dendrograms coincided in splitting the cultivars into two main clusters: a small one, comprising four exotic accessions, and a larger one which could was split into four sub-groups. These were also analyzed on the basis of historical and pedigree information.

The practical approach developed in the study is useful in DNA fingerprinting also. It was found that five varieties could produce unique alleles with four markers, which could be used in identification of these varieties. Of the five varieties, two varieties (Krishnaveni ${ }_{130 b p}$ and Vajram $120 \mathrm{bp}$ ) could be differentiated by a single marker, RM413. Rest of the three varieties could be differentiated by one marker each, RGNMS3876 (Chandana $330 \mathrm{bp}$ ), RGNMS3878 (Sona Mahsuri ${ }_{150 \mathrm{bp}}$ ) and RM3698 (Indursamba ${ }_{200 b p}$ ) (Table 2). The graphical representation of 53 rice varieties based on 23 SSR markers has been depicted in Figure 3. This fingerprinting makes identification and characterization of genotype very easy and further it will be of greater help in background selections during back cross breeding programs. The results in the present study will be useful in understanding molecular variability of the varieties developed from Andhra Pradesh and Telangana and in diversifying the genetic base of rice breeding program, identification of rice varieties in protection of IPR.

\section{References}

Akagi, H., Yokozeki, Y., Inagaki, A., and Fujimura, T. 1997. Highly polymorphic microsatellites of rice consist of AT repeats, and a classification of closely related cultivars with these microsatellite loci. Theor. Appl. Genet. 94: 61-67. 
Berloo, R. V. 2008. GGT 2.0: Versatile software for visualization and analysis of genetic data. J. Heredity. 99(2): 232236.

Cao, T., Duprez, E., Borden, K. L. B., Freemont, P. S., and Etkin, L. D. 1998. Ret finger protein is a normal component of PML nuclear bodies and interacts directly with PML. J. Cell Sci. 111: 1319-1329.

Chakravarthi, K. B., and Naravaneni, R. 2006. SSR marker based DNA fingerprinting and diversity study in rice (Oryza sativa. L). African J. of biotech. 5(9): 684-688.

Deepti, A., Prabhu, K. V., and Singh, A. K. 2011. Analysis of molecular diversity and fingerprinting of commercially grown Indian rice hybrids. J. Plant Biochem. Biotechnol. 21(2): 173-179.

Department of Agricultural Education. Annual Report. 2017-18. Indian Council of Agricultural Research, New Delhi.

Directorate of Economics and Statistics, Govt. of Andhra Pradesh, 2017. (http://desap.cgg.gov.in/home.do).

Directorate of Economics and Statistics, Govt. of Telangana, 2017. (http://ecostat. telangana.gov.in).

Fuentes, J. L., Escobar, F., Alvarez, A., Gallego, G., Duque, M. C., Ferrer, M., Deus, J. E., and Tohme, J. M. 1999. Analyses of genetic diversity in Cuban rice varieties using isozyme, RAPD and AFLP markers. Euphytica. 109(2): 107115.

Gangaprasad, C., Ranjitkumar, N., Malati, S., Deborah, D. A., Abhilash, V., Anuraha, G., Siddiq, E. A., and Lakshminarayana Reddy, V. 2013. Molecular genetic diversity of major Indian rice cultivars over decadal periods. PLoS ONE. 8(6): e66197.

Garris, A. J., Tai, T. H., Coburn, J., Kresovich, S., and McCouch, S. 2005. Genetic structure and diversity in Oryza sativa L. Genetics. 169(3): 1631-1638.

Glaszmann, J. C. 1987 . Isozymes and classification of Asian rice varieties. Theor. Appl. Genet. 74(1): 21-30.

Jain, S., Jain, R. K., and McCouch S. R. 2004. Genetic analysis of Indian aromatic and quality rice (Oryza sativa L.) germplasm using panels of fluorescently-labeled microsatellite markers. Theor. Appl. Genet. 109: 965977.

Khush, G. S. 1995. Modern varieties-Their Real contribution to food supply and equity. Geo. Journal. 35(3): 275-284.

Litt, M., and Luty, J. A. 1989. A hypervariable microsatellite revealed by in vitro amplification of a dinucleotide repeat within the cardiac muscle actin gene. Am. J. Hum. Genet. 44: 397-401.

Lu, H., Redus, M. A., Coburn, J. R., Rutger, J. N., McCouch, S. R., and Tai, T. H. 2005. Population structure and breeding patterns of 145 U.S. rice cultivars based on SSR marker analysis. Crop Sci. 45: 66-76.

McCouch, S. R., Temnykh, S., Lukashova, A., Coburn, J, Declerck, G., Cartinhour, S., Harrington, S., Thomson, M., Septiningsi, E., Semon, M., Moncada, P., and Jiming, L. 2001. Microsatellite markers in rice: Abundance, diversity and applications. In: Rice Genetics IV. IRRI. Manila, Philippines. pp: 117-135.

McCouch, S. R., Teytelman, L., Xu, Y., Lobos, K. B., Clare, K., Walton, M., Fu, B., Maghirang, R., Li, Z., Xing, Y., Zhang, Q., Kono, I., Yano, M., Fjellstrom, R., DeClerck, G., Schneider, D., Cartinhour, S., Ware, D., and Stein, L. 2002. Development and mapping of 2240 new SSR markers for rice (Oryza sativa L.). DNA Res. 9(6): 199-207.

Murray, M. G., and Thompson, W. F. 1980. Rapid isolation of high molecular weight plant DNA. Nucleic Acids Res. 
8: 4321-4325.

Parida, S. K., Dalal, V., Singh, A. K., Singh, N. K., and Mohapatra, T. 2009. Genic non-coding microsatellites in the rice genome characterization, marker design and use in assessing genetic and evolutionary relationships among domesticated groups. BMC Genomics. 10: 140 .

Qian, H. R., Zhuang, J. Y., Lin, H. X., Lu, J., and Zheng, K. L. 1995. Identification of a set of RFLP probes for subspecies differentiation in Oryza sativa L. Theor Appl. Genet. 90: 878-884.

Rohlf, F. J.1998. NTSYS- pc: Numerical taxonomy and multivariate analysis system, version 2.0, Exeter Software. Setauket, New York.

Shobha Rani, N., Srikanth, S., Bhadana, V. P., Jyothi Badri, Sundaram, R. M., Senguttuvel. P., Prasad, G. S. V., Trushar Shah, Prasad, P. V. N. S., Hari Prasad A. S., Subba Rao, L. V., Agarwal, S. K., and Viraktamath B. C. 2014. Genetic diversity and genealogy of rice varieties in India. Directorate of Rice Research, Rajendranagar, Hyderabad-30.

Spada, A., Mantegazza, R., Biloni, M., Caporali, E., and Sala, F. 2004. Italian rice varieties: historical data, molecular markers and pedigrees to reveal their genetic relationships. Plant Breeding. 123: $105-111$.

Tautz, D. 1989. Hypervariability of simple sequences as a general source of polymorphic DNA markers. Nucleic Acids Res. 17(16): 6463-6471.

$\mathrm{Xu}$, Y., Beachell, H., and McCouch, S. R. 2004. Marker-based approach to broadening the genetic base of rice in the USA. Crop Sci. 44:1947-1959.

Yang, G. P., Maroof, M. A., Xu, C. G., Zhang, Q., and Biyashev, R. M. 1994. Comparative analysis of microsatellite DNA polymorphism in landraces and cultivars of rice. Mol. Gen. Genet. 245:187-194.

Yu, S. B., Xu, W. J., Vijayakumar, C, H, M., Ali, J., Fu, B. Y., Xu, J. L., Jiang, Y. Z., Marghirang, R., Domingo, J., Aquino, C., Virmani, S. S., and Li, Z. K. 2003. Molecular diversity and multilocus organization of the parental lines used in the International Rice Molecular Breeding Program. Theor. Appl. Genet. 108: 131-140.

Zhang, Z., Deng, Y., Tan, J., Hu, S., Yu, J., and Xue, Q. 2007. A genome-wide microsatellite polymorphism database for the indica and japonica rice. DNA Res. 14: 37-45.

\section{How to cite this article:}

Balram Marathi and Maliha Allugundu. 2018. Assessment of Genetic Diversity and Fingerprinting of Rice Cultivars of Andhra Pradesh and Telangana with SSR Markers. Int.J.Curr.Microbiol.App.Sci. 7(12): 2553-2563. doi: https://doi.org/10.20546/ijcmas.2018.712.290 